\title{
Identification and Light-Dependent Translocation of a Cone-Specific Antigen, Cone Arrestin, Recognized by Monoclonal Antibody 7G6
}

\author{
Houbin Zhang, ${ }^{1,2}$ Nicolas Cuenca, ${ }^{3}$ Tamara Ivanova, ${ }^{4}$ Jill Church-Kopish, ${ }^{1}$ \\ Jeanne M. Frederick, ${ }^{1}$ Peter R. MacLeish, ${ }^{4}$ and Wolfgang Baehr ${ }^{1,2,5}$
}

Purpose. To elucidate the antigen recognized by monoclonal antibody (mAb) 7G6, a widely used cone-specific marker.

Methods. 7G6 immunocytochemistry was performed on sections of human, primate, and bovine retina. The antigen was immunoprecipitated from human retinal lysates and purified with protein $G$. Edman degradation and liquid chromatography of tryptic peptides combined with tandem mass spectrometry (LC-MS/MS) identified the antigen.

Results. Sequencing of peptides derived from the immunoprecipitated 7G6 antigen identified it as cone arrestin. The identity was confirmed by Western blot analysis with recombinant human cone arrestin and competition with the antibody in immunocytochemistry. Subcellular localization of cone arrestin in dark-adapted and bleached bovine retinas showed that cone arrestin accumulated in cone outer segments of light-adapted retina but was more concentrated in the inner segments of dark-adapted retina. By expression of truncated human cone arrestin mutants systematically deleting areas divergent from bovine and primate cone arrestins, the epitope of $7 \mathrm{G} 6$ was identified as a divergent loop exposed at the surface within the $\mathrm{N}$-domain of cone arrestin.

Concuusions. Several independent methods established that the $7 \mathrm{G} 6$ antigen is cone arrestin. The 7G6 epitope is contained in a divergent loop, the sequence of which is conserved in bovine and primates but not other vertebrate species consistent with the specificity of the antibody. The light-dependent translocation of cone arrestin suggests a role in light-dark adaptation of cones. Because of the location of its gene on the X-chromosome, cone arrestin is a candidate gene for X-linked cone dystrophies. (Invest Ophthalmol Vis Sci. 2003;44:2858-2867) DOI:10.1167/iovs.03-0072

From the ${ }^{1}$ Department of Ophthalmology, Moran Eye Center, and the Departments of ${ }^{2}$ Neurobiology and Anatomy and ${ }^{5}$ Biology, University of Utah, Salt Lake City, Utah; the ${ }^{3}$ Department of Biotechnology, University of Alicante, San Juan de Alicante, Spain; and the ${ }^{4}$ Neuroscience Institute, Department of Anatomy and Neuroscience, Morehouse School of Medicine, Atlanta, Georgia.

Supported by NIH Grant R01EY08123 (WB). Additional support came from the Macular Vision Research Foundation, Research to Prevent Blindness, Inc., and a Center grant from the Foundation Fighting Blindness to the University of Utah. WB is the recipient of a Senior Investigator Award from RPB and a Ralph and Mary Tuck endowment to the Department of Ophthalmology at the University of Utah.

Submitted for publication January 22, 2003; revised March 14, 2003; accepted March 17, 2003

Disclosure: H. Zhang, None; N. Cuenca, None; T. Ivanova, None; J. Church-Kopish, None; J.M. Frederick, None; P.R. MacLeish, None; W. Baehr, None

The publication costs of this article were defrayed in part by page charge payment. This article must therefore be marked "advertisement" in accordance with 18 U.S.C. $\$ 1734$ solely to indicate this fact

Corresponding author: Wolfgang Baehr, University of Utah Health Science Center, Moran Eye Center, 75 N. Medical Drive, Salt Lake City, UT 84112; wbaehr@hsc.utah.edu.
The cone-specific monoclonal antibody (mAb) 7G6 has 1 been shown to recognize all cone subtypes in the adult primate retina and the labeling for it has been observed throughout the cytoplasm of the cones. ${ }^{1}$ The antibody 7 G6 has been used as a universal primate cone marker in normal and degenerating retina in several subsequent publications. ${ }^{2,3}$ (A teaching set on normal and diseased human retinas assembled by Ann Milam displaying retina sections stained with mAb 7G6 is available at http://www.penneye.com/html/jeff_berger_ memorial.html.) The 7G6 antigen was recently shown to colocalize in the cone cytoplasm with an unidentified $\mathrm{p} 45$ protein that appears to share an epitope with the transcription factor NRL. ${ }^{4}$ During early primate development, some heterogeneity in 7G6 labeling was observed. For example, a subset of blue cones was delayed in their acquisition of 7G6 immunoreactivity, suggesting a delayed expression of the antigen, whereas the onset of $7 \mathrm{G} 6$ immunoreactivity in red and green cones preceded both the expression of cone opsins and the formation of synaptic contacts in the inner plexiform layer. ${ }^{1}$

To investigate the nature of the antigen and its gene more closely, we first used Western blot analysis of human macula components to determine the mobility of the antigen in SDSPAGE. We then purified the antigen, generated proteolytic peptides and sequenced them by both Edman degradation and liquid chromatography combined with mass spectrometry (LCMS/MS). Sequence analysis of multiple fragments showed unambiguously that the 7G6 antigen is indistinguishable from cone arrestin (cArr, or X-arrestin). (Zhang $\mathrm{H}$, Cuenca $\mathrm{N}$, Church-Kopish J, Ivanova T, Frederick JM, MacLeish PR, Baehr W, ARVO abstract 1414, 2002). Arrestins are a large gene family involved in regulation of G-protein-coupled receptors and G-protein-linked cascades. The cone's visual cascade is thought to function very similarly to that of rods, but is composed of components encoded by distinct genes. Most of these components have been cloned and characterized, including cone pigments, ${ }^{5}$ cone transducin $\alpha$-subunit, ${ }^{6}$ cGMP PDE $\alpha^{\prime},{ }^{7}$ and cyclic nucleotide gated channel $\alpha(\mathrm{CNG} 3)^{8}$ and $\beta$ subunit. ${ }^{9}$ Most regulatory components such as guanylate cyclase-activating protein (CGAP)-1, ${ }^{10}$ guanylate cyclase (GC)-1, ${ }^{11}$ RGS9-1, ${ }^{12}$ G-protein-coupled receptor kinase (GRK)-1, ${ }^{13}$ and recoverin $^{14,15}$ are present in both rods and cones in mammals. Exceptions are the arrestins, which occur in cell-specific isoforms.

One hallmark of arrestin function is its relocalization in the rod cell in a light-dependent manner. In dark-adapted retina, arrestin is located in the inner segment, but redistributes rapidly to the outer segment on illumination. ${ }^{16,17}$ Recently, cone arrestin was shown to translocate in the mouse retina, depending on illumination. ${ }^{18}$ We found that bovine cone arrestin redistributes similarly in light- and dark-adapted bovine retina. In the light, cone arrestin accumulates in the cone outer segments, presumably interacting with bleached cone visual pigments and quenching the cone cascade. In contrast, in the dark, cone arrestin accumulates in the inner segment. The light-dependent translocation of cone arrestin depending on 
light history, similar to that of rod arrestin, suggests a novel mechanism of light- dark adaptation in cones.

\section{Materials ANd Methods}

\section{Immunoprecipitation and Peptide Sequencing}

Retinal extracts were prepared from human macular buttons obtained from the Lions Eye Bank at the University of Utah 4 to 8 hours after death. A crude extract of human maculae was mixed with $7 \mathrm{G} 6$ antibody and protein-G-coupled agarose (Santa Cruz Biotechnology, Santa Cruz, CA) in IP buffer (20 mM Tris [pH 7.5], $150 \mathrm{mM} \mathrm{NaCl}$, and $0.1 \%$ Tween 20). The mixture was incubated 2 hours at room temperature on a rocking platform. The agarose beads were pelleted by brief centrifugation and washed three times with IP buffer. SDS $(2 \times$ gel loading buffer $(45 \mu \mathrm{L})$ was added to the agarose beads followed by boiling for 2 minutes to elute the bound proteins completely. The proteins were fractionated by $10 \%$ SDS-PAGE gel and stained with Coomassie blue dye. Stained bands corresponding to the antigen were excised from SDS-PAGE gel and submerged into destaining solution $\left(0.2 \mathrm{M} \mathrm{NH}_{4} \mathrm{HCO}_{3}\right.$ containing $30 \%$ acetonitrile). After incubation at $30^{\circ} \mathrm{C}$ for 1 hour, the liquid was removed, and the fresh destaining solution was added. The process was repeated until the gels were completely destained. The gels were then fully dehydrated in a concentrator (SpeedVac; Thermo Savant, Holbrook, NY). For in-gel digestion, the dried gels were rehydrated with $40 \mu \mathrm{L} 0.2 \mathrm{M} \mathrm{NH}_{4} \mathrm{HCO}_{3}$ containing $0.4 \mu \mathrm{g}$ sequencing-grade trypsin (Promega Corp., Madison, WI). The proteolysis was stopped by adding $1 / 10$ volume of $10 \%$ trifluoroacetic acid (TFA). The released peptides were extracted several times from the gel with $100 \mu \mathrm{L}$ of $0.1 \%$ TFA and $60 \%$ acetonitrile followed by concentration (Speed-Vac; Thermo Savant) concentration. The mixed peptides were submitted to the peptide-sequencing core facility in University of Utah for separation by HPLC and sequencing by Edman degradation. Meanwhile, the peptide mixture prepared under the same conditions was submitted to the mass spectrometer core facility (University of Utah) and analyzed by LC/MS-MS. The tryptic peptides were loaded onto an HPLC system linked to a mass spectrometer (LCQ Deca; Thermo Finnigan, San Jose, CA). The peptides were eluted into the mass spectrometer and MS/MS spectra were generated. The spectra were subjected to search against the databases on computer with a commercially available program (Sequest Software, Lisle, IL) to find the matched peptides. The retrieved peptide sequences were compared with the sequences in GenBank (http://www.ncbi. nlm.nih.gov/Genbank; provided in the public domain by the National Center for Biotechnology Information, Bethesda, MD).

\section{Western Blot Analysis}

Human maculae were isolated from freshly obtained retinas using a trephine ( $6 \mathrm{~mm}$ diameter). Two maculae were dropped into a microcentrifuge tube and homogenized with a plastic pestle in $100 \mu \mathrm{L}$ hypotonic buffer A (20 mM Tris, [pH 8.0], 1 mM EDTA, 1 mM dithiothreitol [DTT], and $100 \mu \mathrm{M}$ phenylmethylsulfonyl fluoride [PMSF]). The tissue lysate was centrifuged at $600 \mathrm{~g}$ for 5 minutes at $4^{\circ} \mathrm{C}$. The supernatant was removed and saved, and the pellet was homogenized again in buffer $\mathrm{A}$ and recentrifuged as before. The supernatants from these two centrifugations were combined and subjected to ultracentrifugation at $250,000 \mathrm{~g}$ for 1 hour at $4{ }^{\circ} \mathrm{C}$. One tenth of the supernatant and the entire pellet from ultracentrifugation were subjected to $10 \%$ SDS-PAGE gel electrophoresis, and the resolved polypeptides were transferred to a nitrocellulose filter (Bio-Rad, Hercules, CA). The filter was probed with mAb 7G6 (1:150) followed by horseradish peroxidase [HRP]-conjugated secondary antibody. The signal was visualized using phosphorescence (ECL system; NEN Life Science, Boston, MA) and detected by x-ray film.

\section{Immunocytochemisty and Preabsorption Assay}

Monkey eyes were obtained from the Texas Primate Center (Alice, TX) and fixed as described previously. ${ }^{19}$ Adult human and bovine eyes were dissected to remove the anterior segment, and the eye cup was transferred to freshly prepared $4 \%$ paraformaldehyde in $0.1 \mathrm{M}$ phosphate buffer ( $\mathrm{pH} 7.4)$. After fixation for 2 hours at room temperature, the eye cup was immersed in $10 \%$ sucrose in $0.1 \mathrm{M}$ phosphate ( 1 hour), $20 \%$ sucrose in $0.1 \mathrm{M}$ phosphate (1 hour), and 30\% sucrose in $0.1 \mathrm{M}$ phosphate buffer (overnight). A small piece of retina-RPE was isolated with a razor blade, embedded in commercial medium (TBS; Triangle Biomedical Sciences, Durham, NC) and frozen. Sections (12- $\mu \mathrm{m}$ thick) were cut with a cryostat and mounted on gelatin-coated or charged slides (Superfrost/Plus, Fisher Scientific, Pittsburgh, PA). Bovine sections from light- and dark-adapted specimens were mounted pair-wise so that tissues from both were incubated in the same pool of antibody solution. This facilitated direct comparison of 7G6 immunolocalization correlating with light versus dark adaptation.

For immunocytochemistry, the sections were blocked with $0.1 \mathrm{M}$ phosphate buffer ( $\mathrm{pH} 7.4$ ), containing $1 \% \mathrm{BSA}, 0.1 \%$ Triton $\mathrm{X}-100$, and $10 \%$ normal goat serum, and probed by $7 \mathrm{G} 6$ antibody (1:100 or 1:200 dilution) followed by FITC-conjugated goat anti-mouse or TRITC-conjugated goat anti-mouse secondary antibody. For double labeling, the sections were incubated simultaneously with mAb $7 \mathrm{G} 6$ and polyclonal antibodies anti-calbindin (Swant, Bellinzona, Switzerland) overnight at $4^{\circ} \mathrm{C}$. Sections were visualized by application of rhodamine (TRITC)conjugated goat anti-mouse and FITC-conjugated goat anti-rabbit secondary antibodies. The immunolocalization was imaged using a confocal microscope (model LSM 510; Carl Zeiss Meditech, Thornwood, NY) set to an optical slit of $1 \mu \mathrm{m}$.

A preabsorption study was performed to verify $7 \mathrm{G} 6$ antibody specificity. Fresh human retina was obtained from a 4-month-old infant whose eye was enucleated because of retinoblastoma (biopsy tissue provided by Nick Mamalis, University of Utah). The nontumorous portion of the retina-RPE was dissected 2.5 hours after enucleation, fixed for 2 hours in $4 \%$ paraformaldehyde in $0.1 \mathrm{M}$ phosphate buffer ( $\mathrm{pH} 7.4$ ), and cryoprotected in sucrose. Frozen sections were blocked for 1 hour with $10 \%$ normal goat serum before incubation with $\mathrm{mAb}$ 7G6. As a negative control, 7G6 (1:200) was preabsorbed for 6 hours with an excess amount of recombinant human cone arrestin $(\sim 15 \mu \mathrm{g}$ rCArrHis $/ 50 \mu \mathrm{L}$ solution) before the solution was applied to the sections of retina. Immunoreactive sites were visualized by incubation 1 hour in FITC-conjugated goat anti-mouse IgG (1:300, cat. \#115-095146; Jackson ImmunoResearch, West Grove, PA) as the secondary antibody and imaged as described for immunocytochemistry.

\section{Expression and Purification of Human His-Tagged Cone Arrestin}

The human His-tagged cone arrestin (hcArrHis) expression construct was kindly provided by Cheryl Craft (University of Southern California). For this construct, full-length human cone arrestin cDNA was cloned in frame into an expression vector (pTRC-His B; Promega). The plasmid was transformed into Escherichia coli ER2556 (New England Biolabs, Boston, MA), and a single colony was inoculated into $5 \mathrm{~mL}$ Luria-Bertani (LB) medium and grown at $37^{\circ} \mathrm{C}$. The overnight culture was diluted into $100 \mathrm{~mL}$ of and continued growing to an $\mathrm{OD}_{600}$ of 0.6 to 0.7 . Isopropyl- $\beta$-D-1-thiogalactopyranoside (IPTG) was added to the culture to a final concentration of $1 \mathrm{mM}$ to induce the expression of recombinant protein. After 5 hours of induction at $37^{\circ} \mathrm{C}$, the culture was harvested. The cells equal to $1.5 \mathrm{~mL}$ of culture were lysed with lysozyme, and a fraction of the lysate was analyzed by Western blot with 7G6 antibody. Recombinant hcArrHis was purified from $500 \mathrm{~mL}$ of culture with a HisTrap column (Pharmacia/Amersham, Piscataway, NJ) according to the manufacturer's instructions. The purified protein was verified by SDS-PAGE gel and quantified using a protein assay kit (Cat. no. 500-0006; Bio-Rad).

\section{Mutagenesis}

To prepare human cone arrestin deletion mutants, mutagenesis was performed with a kit (QuickChange; Stratagene, LaJolla, CA). Five deletion mutants (see Fig. 4A) introducing stop codons after codons 
$97,168,187,343$, and 355 were generated. Briefly, two complementary primers harboring a nonsense mutation in the center corresponding to the desired stop position were synthesized (DNA synthesizer model 392; Applied Biosystems, Foster City, CA). Simultaneously, a silent restriction site $(X b a \mathrm{I})$ was introduced into the primers to test the newly generated plasmid DNAs for successful mutagenesis by XbaI restriction digest. The primers were mixed with $50 \mathrm{ng}$ of template plasmid and recombinant $P f u$ polymerase, and the amplification was performed for 16 cycles according to manufacturer's instructions. Methylated template DNA was removed by digestion with $D p n \mathrm{I}$. A small aliquot of the reaction mixture was used to transform $E$. coli. The mutation was confirmed by DNA sequencing (Prism capillary sequencer; Applied Biosystems). The IPTG-induced expression, purification of the truncated proteins and Western blot were performed, as described earlier.

\section{Determination of Arrestin Gene Structures}

Contigs from human (AL357752), mouse (AC091784), rat (AC095218), and zebrafish (AL590151) were identified by screening the National Center for Biotechnology Information (NCBI) high-throughput gene sequences (htgs) using cone arrestin protein sequences and tblastn (NCBI database). Gene structures were determined with cDNA or protein sequences as templates. The novel zebrafish cArr gene structure was derived using 15 zebrafish cArr expressed sequence tags (ESTs)-AW826248, AW826514, BE015717, BE015912, BE016049, BF937662, BF937816, BF938932, BG305632, BG306321, BG306785, BG306798, BI706094, BI879022, and BI879162-covering the entire mRNA from the transcription start point to the poly(A) tail. Splice variations were examined in all genes using partial cArr-specific ESTs. Software for DNA analysis was Omiga 2.0 (Accelrys, Cambridge, UK; available at http://www.accelrys.com/dstudio/ds_gene/) and DNA * (DNAstar, Inc., Madison, WI; http://www.dnastar.com), both available commercially.

\section{Light and Dark Adaptation of Bovine Eyes}

A pair of freshly enucleated bovine eyes was obtained from a local abattoir. One eye was dark adapted for 3 hours on ice in a light-tight metal can sealed with black tape and dissected in a darkroom under dim red light. The retina-RPE was fixed in the dark with $4 \%$ paraformaldehyde solution. The other eye, placed on ice, was kept at room light with an intensity of approximately 500 lux (normal room light). The eye was dissected in normal room light, and the retina-RPE was fixed in $4 \%$ paraformaldehyde and processed for immunocytochemistry, as described earlier.

\section{RESUlTS}

\section{Specificity of $\mathbf{m A b} 7 \mathrm{G} 6$}

We first explored the cell specificity of the mAb 7G6 in human, bovine, and primate retina (Fig. 1). Staining human frozen sections with 7 G6 (Fig. 1B) reveals expression of the antigen exclusively in cones, apparently distributed throughout the cell including cone pedicles, somata, and inner and outer segments. Human retina incubated with a polyclonal anti-calbindin antibody (Fig. 1A) reveals labeling in the cytoplasm of the long-wavelength (LW) cone inner segments, the cell bodies, and the synaptic pedicles. Staining in the outer segments with this antibody is relatively weak. Note that short-wavelength (SW) cones are only very faintly labeled with anticalbindin. The identity of these cones as SW cones was recently confirmed by double labeling with anti-blue opsin antibody. ${ }^{20}$ Anti-calbindin also labeled bipolar cells which are 7G6 immunonegative (results not shown). The function of calbindin, an $\mathrm{EF}$ hand containing small $\mathrm{Ca}^{2+}$ binding protein with widespread distribution in neurons, is unknown, but it has been suggested that it transports intracellular $\mathrm{Ca}^{2+}$ and protects against $\mathrm{Ca}^{2+}$ mediated apoptosis. ${ }^{21}$ When human retina sections were double labeled with anti-calbindin and 7G6 (Fig. 1C), synaptic pedicles and inner segments of LW cones, but not SW cones, showed colocalization. Apart from human (Figs. 1B, 1C) and monkey (Fig. 1E) retinas, only bovine cones were found to be immunopositive with mAb 7 G6 (Fig. 1D). Labeling of other vertebrate retinas (mouse, ground squirrel, salamander) with 7G6 was unsuccessful (results not shown), indicating that the 7G6 epitope is only present in primate and bovine antigens. The results are consistent with mAb 7G6 recognizing an antigen present nearly exclusively in the cytoplasm of primate and bovine cone photoreceptors. Uniform labeling with 7G6 and variation in double labeling with anticalbindin suggests that the 7G6 antigen is present in all cone subtypes.

\section{Identification of the 7G6 Antigen}

To identify the 7G6 antigen in cones, we used Western blots with macular extracts from human retina (the macula is rich in cone photoreceptors). Western analysis revealed the presence of the 7G6 antigen exclusively in the hypotonic supernatant, and its absence in the membrane pellet (Fig. 2A). The apparent mobility in denaturing SDS gels is approximately 44 to $46 \mathrm{kDa}$, independent of denaturing the extract at high or low temperatures. To immunoprecipitate the $7 \mathrm{G} 6$ antigen, the hypotonic macular extract was incubated with $\mathrm{mAb} 7 \mathrm{G} 6$ and protein-Gcoupled agarose. ${ }^{22}$ The immunoprecipitate was analyzed by SDS-PAGE (Fig. 2B) revealing IgG (Fig. 2B, arrow 1) and the 7 G6 antigen (Fig. 2B, arrow 2). We then determined proteolytic peptide sequences of the immunoprecipitated 7G6 antigen by two independent methods: Edman degradation and LC-MS/MS. Edman degradation of a single proteolytic polypeptide yielded a sequence identical with a short fragment of human cone arrestin (cArr) at positions 166-175 (Fig. 2C). Separation of a mixture of proteolytic fragments by liquid chromatography and subsequent mass spectrometric analysis of individual peaks allowed identification of fragments covering essentially the entire cArr polypeptide, except for the $\mathrm{N}$-terminal region (Fig. 2C). The results show complete identity of the 7G6 antigen with human cArr, a polypeptide of 388 amino acid residues. The calculated molecular mass of human cArr is $43 \mathrm{kDa}$, consistent with the apparent mobility of the 7 G6 antigen ( $44-48 \mathrm{kDa}$, Fig. 2A). In control experiments, we expressed His-tagged human cArr in bacteria and analyzed the recombinant protein by Western blot analysis (Fig. 2D). The results show that the recombinant protein has an apparent mobility of $50 \mathrm{kDa}$, slightly larger than the 7G6 antigen (the recombinant protein carries a His tag), and is immunopositive with mAb 7G6. When the mAb 7G6 is preabsorbed with recombinant cArr, immunohistochemical labeling of cones with 7 G6 is completely abolished (Fig. 2E). These results established unambiguously that the $7 \mathrm{G} 6$ antigen is cone arrestin.

\section{Light-Dependent Redistribution of Cone Arrestin}

The identification of the 7G6 antigen as cone arrestin raised the interesting question of whether cone arrestins redistribute in cones, as has been observed for rod arrestin (S-antigen) and other phototransduction components (for review, see Ref. 23). In dark-adapted mammalian retina, arrestin is located in the inner segment, but moves rapidly to the outer segment, on illumination. This apparent movement was controversial for many years, but recently was shown to occur unambiguously with rod $\mathrm{T} \alpha .{ }^{24}$ Because dark-adapted primate retinas are very difficult to obtain, we performed a series of experiments with light- and dark-adapted bovine retinas, the only mammalian species besides primates with 7 G6 immunoreactivity (Fig. 1D). In dark-adapted bovine retinas, cone arrestin distributes 
Figure 1. Specificity of mAb 7 G6 in human, bovine, and monkey retina. (A) Labeling of human cone photoreceptors with a commercially available polyclonal anti-calbindin antibody. Calbindin immunolocalization is indicated by green labeling (FITCconjugated secondary antibody). The brightly labeled cones are LW cones, and the faintly labeled cones are SW cones. ${ }^{20}$ Calbindin was present in cone pedicles and the cytoplasm of cone inner segments and at lower levels in the outer segments. (B) Labeling of human cone photoreceptors with 7G6 antibody visualized with rhodamine-conjugated secondary antibody. Note uniform labeling of LW and SW cones. (C) Double labeling with anti-calbindin and 7G6 antibodies. Colocalization of calbindin and 7G6 antigen was strongest in the inner segments and synaptic pedicles of LW cones. (D) Bovine sections of retina were labeled with $7 \mathrm{G6}$ (red). As in humans, all of the cone cells including synaptic pedicles labeled with 7G6. (E) Macaque retinas were labeled with FITC-conjugated secondary antibody. All retinas were fixed under normal room light, and no precautions for dark adaptation or complete bleaching were taken.
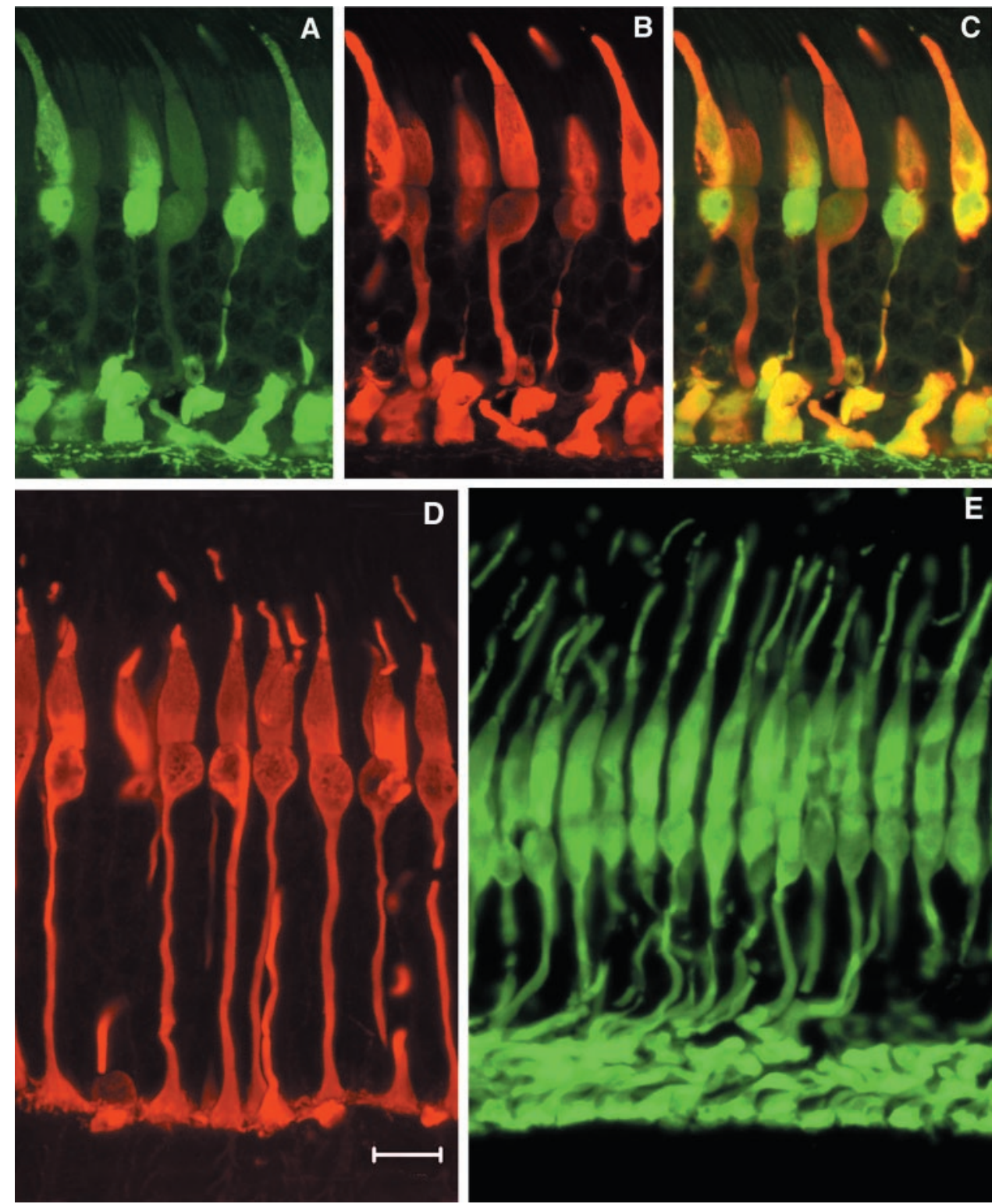

throughout the cytoplasm of cones, but accumulates predominantly in the myoid region of cone inner segments (Fig. 3A), the region where biosynthesis takes place. In the light, cone arrestin accumulates strongly in the outer segments (Fig. 3B), presumably interacting with bleached cone visual pigments and inactivating the cone cGMP cascade. Accumulation of these components in distinct compartments of photoreceptors suggests the presence of a novel mechanism of photoreceptor light- and dark-adaptation in both rod and cone photoreceptors.

\section{Cone Arrestin Genes}

To investigate potential links to human retina disease and the diversity of cone arrestins, we next analyzed vertebrate cone arrestin gene structures. The human and mouse cone arrestin genes have been cloned, and their exon-intron arrangement has been determined. ${ }^{25,26}$ These genes were shown to consist of 17 exons (one noncoding exon is located in the 5'untranslated region [UTR]). We identified human (AC068231, AL357752) and mouse (AC091784) genomic contigs in GenBank that verified the published gene structures (Figs. 4A, 4B).
The human contig AL357752 containing the entire cArr gene localized the gene to chromosome $X$ (q13.2-21.1) which significantly narrowed the published locus (Xcen-Cq22). ${ }^{25}$ In addition to human and mouse, we determined the gene structures of rat (Rattus norvegicus) and zebrafish (Danio rerio) cArr from large anonymous contigs (AC095218, AL590151) containing the entire cArr genes. As expected, the rat cArr gene structure (Fig. 4C), consisting of 17 exons, was nearly identical with that of mouse and human (Figs. 4A, 4B). In contrast, the zebrafish cArr gene (Fig. 4C) consisted of only 16 exons and encoded a 362 amino acid polypeptide with a significantly truncated C-terminal region. The zebrafish cArr gene features an exon 15 that is only $4 \mathrm{bp}$ (one amino acid) in length, separated from exons 14 and 16 by a 302 and 91 bp introns, respectively. Like the mammalian cArr genes, the first exon is located in the $5^{\prime}$ UTR. The predicted RNA and amino acid sequences were verified by 15 independent ESTs (10 of which are shown in Fig. 4D) that cover the entire 5'UTR, the coding sequence, and the $3^{\prime}$ UTR. In contrast to complex alternative splicing observed in human and mouse cArr genes, ${ }^{25,26}$ the zebrafish ESTs deposited in GenBank show no significant 


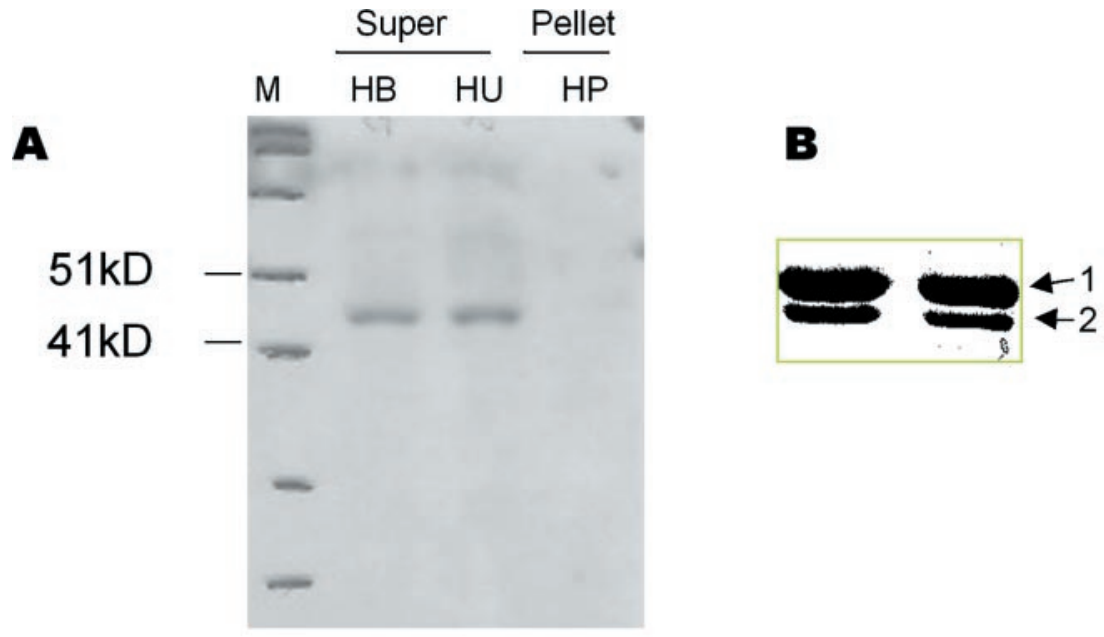

C

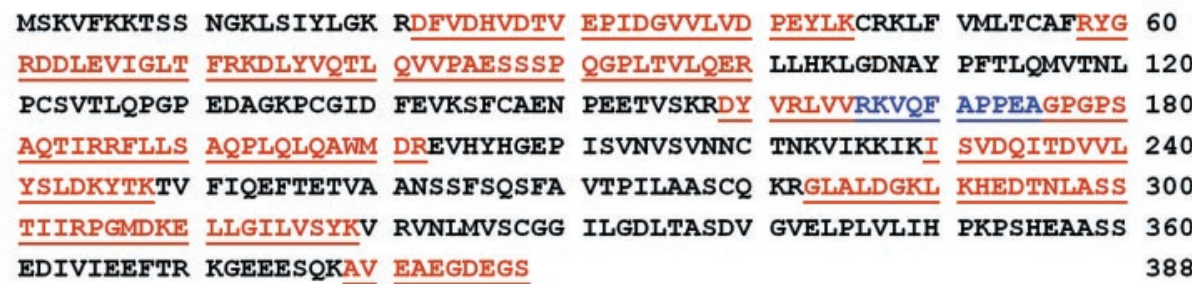

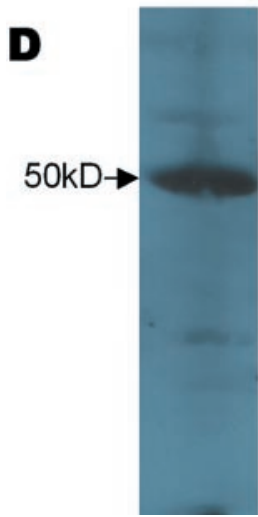

$\mathbf{E}$
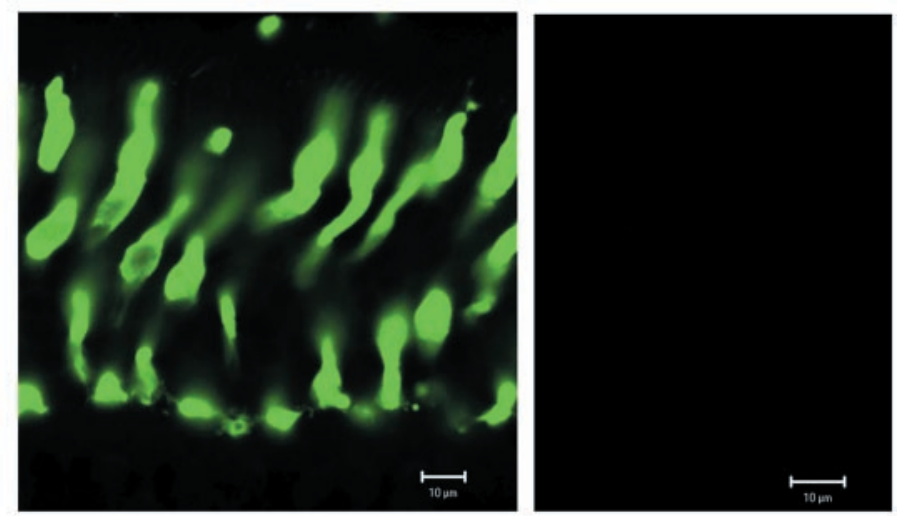

FIGURE 2. Identification of the 7G6 antigen. (A) SDS-PAGE ( $10 \%$ polyacrylamide gel) followed by Western blot of hypotonic human macula extract probed with mAb 7G6. The membrane fraction was separated from soluble proteins by ultracentrifugation. The antigen was detected by Western blot with 7 G6 antibody. HB, homogenate supernatant boiled; HU, homogenate supernatant unboiled. HP, homogenate pellet. (B) Immunoprecipitation of antigen with $\mathrm{mAb}$ 7G6. The human macular crude extract obtained after ultracentrifugation was incubated with mAb 7G6 and protein G-coupled agarose. The immunoprecipitate was analyzed by SDS-PAGE. Arrow 1: IgG; arrow 2: 7G6 antigen. (C) Sequence determination of proteolytic peptides. The peptide sequences unambiguously match human cone arrestin. Peptide sequences identified by Edman degradation are underscored in blue, and sequences identified by LCMS/MS are underscored in red. (D) Western blot of bacterial recombinant His-tagged human cone arrestin. The bacterial pellet was lysed by sonication, and the soluble fraction was probed with mAb 7G6. The immunopositive antigen has a mobility of $\sim 50 \mathrm{kDa}$ (arrow). (E) Competition of mAb $7 \mathrm{G} 6$ with recombinant human cone arrestin. Left: human 4-month-old retinoblastoma retina was probed with mAb 7G6 (1:100 dilution) overnight at $4^{\circ} \mathrm{C}$. Right: mAb 7 G6 preabsorbed 6 hours with $15 \mu \mathrm{g}$ recombinant cone arrestin and incubated overnight as in (A). Recombinant cone arrestin abolished the native cone arrestin. variation in splicing (apart from one EST with a short deletion in the $\left.3^{\prime} U T R\right)$. Phylogenetic analysis of the polypeptide sequences reveals that rod and cone arrestins fall into two distinct, but closely related subfamilies, as has been shown previously. ${ }^{27}$ The $7 \mathrm{G} 6$ epitope, however, is only present in primate and bovine cone arrestins.

\section{Functional and Divergent Domains in Cone Arrestins}

Vertebrate rod and cone arrestin amino acid sequences are highly conserved (Fig. 5) suggesting that the overall threedimensional structure cone arrestin is very similar to that of rod arrestin. The $0.28-\mathrm{nm}$ crystal structure of rod arrestin showed a bipartite molecule consisting of $\mathrm{N}$ - and C-domains. ${ }^{28}$ The sequence similarity among rod arrestins is $51 \%$ to $83 \%$ (compared with bovine arrestin), among cone arrestins $52 \%$ to $84 \%$ (compared to bovine cone arrestins). Bovine arrestin is $47 \%$ to $55 \%$ similar to all cone arrestins shown in Figure 5. The arrestin signature region (positions 57-75 in human cArr) is essentially unaltered among all arrestins. Two N-linked glycosylation sites
(Fig. 5, gray boxes) are conserved in the rod and cone arrestins, but N-glycosylation has not been established in arrestins (one report $^{29}$ suggested that a small population of rod arrestin may be glycosylated). Among vertebrate cone arrestins, several regions are divergent, the $\mathrm{C}$-terminal region (last 30 residues) and three relatively short regions of approximately 10 to 20 residues in the N-terminal half and the central part of the molecule (Fig. 5, bars 1-4). Region 3 in cone arrestin is analogous to a linker connecting two $\beta$ sheets in the $\mathrm{N}$ - and C-domains of arrestin, respectively. In rod arrestin, the C-terminal region is important for locking arrestin into an inactive state. ${ }^{28}$ Sequence divergence in this region suggests flexibility in the length to exert this important function.

The amino acid sequences in Figure 5 are from the following sources: hum_cArr, accession no. 13633229 (National Institutes of Health); bov_cArr, accession no. D85340 ${ }^{36}$; mus_cArr, accession no. NP_573468; rat cArr, derived from contig AC095218; xen_cArr, accession P5148327; zebArr3 (Danio rerio), derived from a cDNA sequence extracted from an anonymous genomic contig, accession no. AL590151 (Sanger 


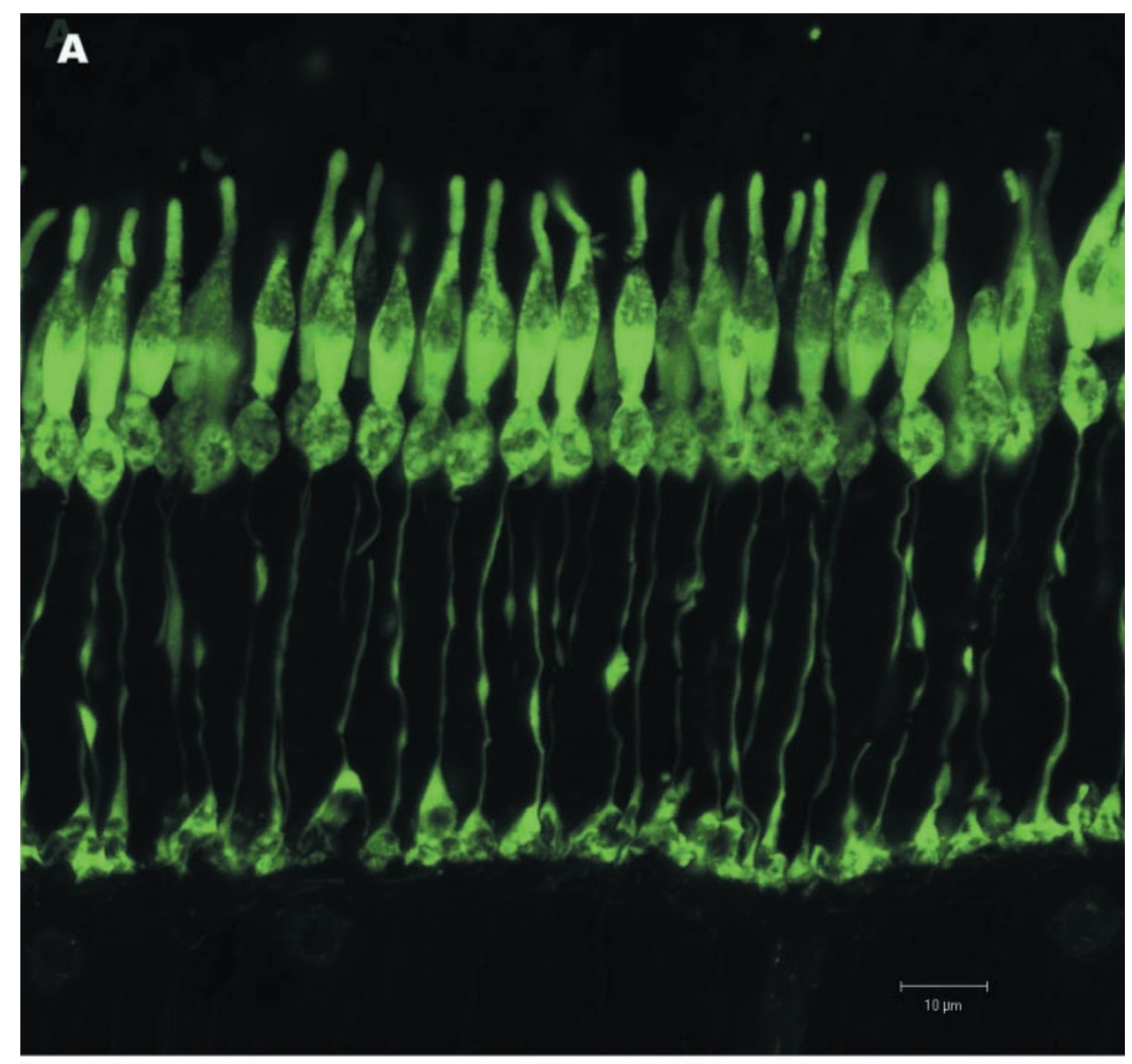

B

Figure 3. Redistribution of cone arrestin in bovine cones, depending on the light history. (A) Dark-adapted bovine retinas. mAb 7G6 labeled the synaptic pedicles, the somata, and the inner and outer segments of the cones. In the dark, the bulk of cone arrestin was concentrated in the myoid region, the place of biosynthesis. (B) Fully bleached bovine retina. The bulk of cone arrestin was in the outer segments, where phototransduction occurs. The confocal images shown are representative of experiments performed with three different pairs of eyes in the fall of 2001 and the spring and summer of 2002 . 

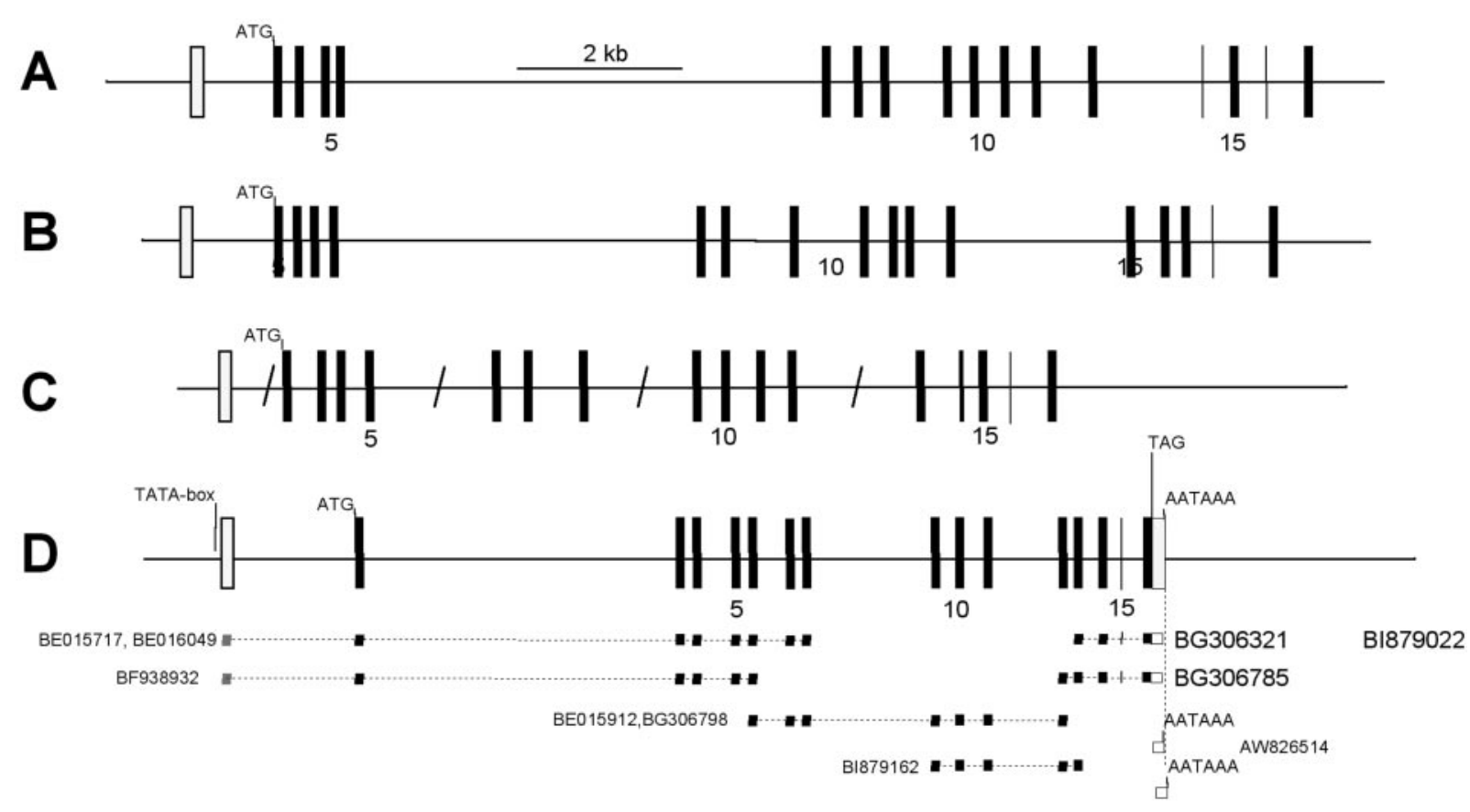

FiguRE 4. Gene structures of vertebrate cone arrestins. The exon-intron arrangements were determined from publicly available anonymous contigs deposited in GenBank: human cArr contig, AL357752, containing a complete human cArr DNA sequence on chromosome X (q13.2-21.1; length, 178,868 bp); mouse cArr contig AC091784, length 215,734 bp; rat cArr contig, AC095218, 73 unordered pieces (length, 183,219 bp); and zebrafish cArr contig AL590151, in ordered pieces, length 136,930 bp. Several independent zebrafish ESTs, shown below the gene diagram, are consistent with the predicted RNA/amino acid sequences of zebrafish cArr. Three ESTs extend presumably to the transcription start point at the beginning of the 5'UTR exon. Two 3'UTR ESTs carry poly(A) tails. A composite zebrafish cArr mRNA sequence from the transcription start point to the beginning of the poly(A) tail has been deposited in GenBank. Black boxes: coding exons; gray boxes: noncoding exons.

Centre, Hinxton Hall, UK); bov_Arr, bovine arrestin (accession AAA30377); mus_Arr, mouse arrestin (accession no. NP_03314); and hum_arr, human arrestin (accession no. 14737493). The sequences were aligned with Clustal W (http://www.ebi.ac.uk/ clustalw/ provided in the public domain by the European Bioinformatics Institute, European Molecular Biology Laboratory, Heidelberg, Germany) and shaded with Boxshade (http://www. ch.embnet.org/software/box_form.html/ provided in the public domain by the Ludwig Institute of Cancer Research and the ISREC Bioinformatics Group, Swiss Institute of Bioinformatics, Basel, Switzerland).

\section{Identification of the mAb 7G6 Epitope}

To determine the epitope for 7G6 in human cone arrestin, we systematically deleted the divergent regions, starting at the C-terminal end, and assayed the truncation mutants for 7G6 immunoreactivity by Western blot analysis. Because human and bovine cArr $\mathrm{C}$ termini are nearly identical and distinct from the $\mathrm{C}$ termini of other cone arrestins, we suspected that the epitope for mAb 7G6 may be located there, particularly because this region carries a number of charged residues and is predicted to be antigenic by exposure on the surface of the molecule (see Fig. 2 in Ref. 28). However, C-terminal deletion mutants in which 23 and $35 \mathrm{C}$-terminal residues were removed by site directed mutagenesis ( $\Delta 4$ and $\Delta 5$ in Fig. 5), were still immunoreactive (Fig. 6, bottom). We then deleted residues 188-388 ( $\Delta 3$ in Fig. 5), 169-388 ( $\Delta 2)$, and 98-388 ( $\Delta 1)$ in cone arrestin, and only deletion mutant $\Delta 1$ was shown to be immunonegative with 7 G6 (Fig. 6, top). Inspection of the sequence alignment narrowed the antigenic determinant for $7 \mathrm{G} 6$ in human cArr to a short region in the $\mathrm{N}$-domain. This region is nearly identical in human and bovine cArr, but divergent in rodent and other vertebrate cone arrestin sequences. We con- clude that the epitope recognized by mAb $7 \mathrm{G} 6$ is located in this divergent loop exposed to the surface of cone arrestin.

\section{Discussion}

We have shown that a widely used immunocytochemical cone marker recognizes cone arrestin, a regulatory component of the cone cascade. Using deletion mutants of human cone arrestin, we have shown that the antigenic determinant is located in a divergent surface-exposed loop in the N-domain of cone arrestin, assuming that cone arrestin has a three-dimensional structure that is very similar to rod arrestin. The high sequence conservation between rod and cone arrestins (Fig. 5) largely justifies this conclusion. The proposed antigenic determinant is nearly identical in bovine and human cone arrestin, but is divergent in other cone arrestin sequences, consistent with the presence of the 7G6 epitope only in human, primate, and bovine retinas.

The function of arrestins consists of desensitizing a G-protein-coupled receptor (in photoreceptors, the rod and cone pigments). Similar to rod arrestin (for review, see Ref. 30), the function of cone arrestin is presumed to consist of interacting with phosphorylated cone visual pigments and downregulating or quenching the cone cascade, thus contributing to desensitization and light adaptation. ${ }^{31}$ Regeneration of the rod and cone visual pigments is thought to require phosphorylation by a rhodopsin kinase termed GRK1 $1^{32}$ or by both GRK1 and GRK $7.33,34$

Recombinant cone arrestins show little affinity for phosphorylated or nonphosphorylated rhodopsin..$^{35,36}$ In vitro work with recombinant mouse cone arrestin suggests that it interacts with chicken cone pigments in a light- and phosphorylation-dependent manner. ${ }^{18}$ Based on crystal structure data of rod arrestin, it is assumed that the receptor's C-terminal tail 


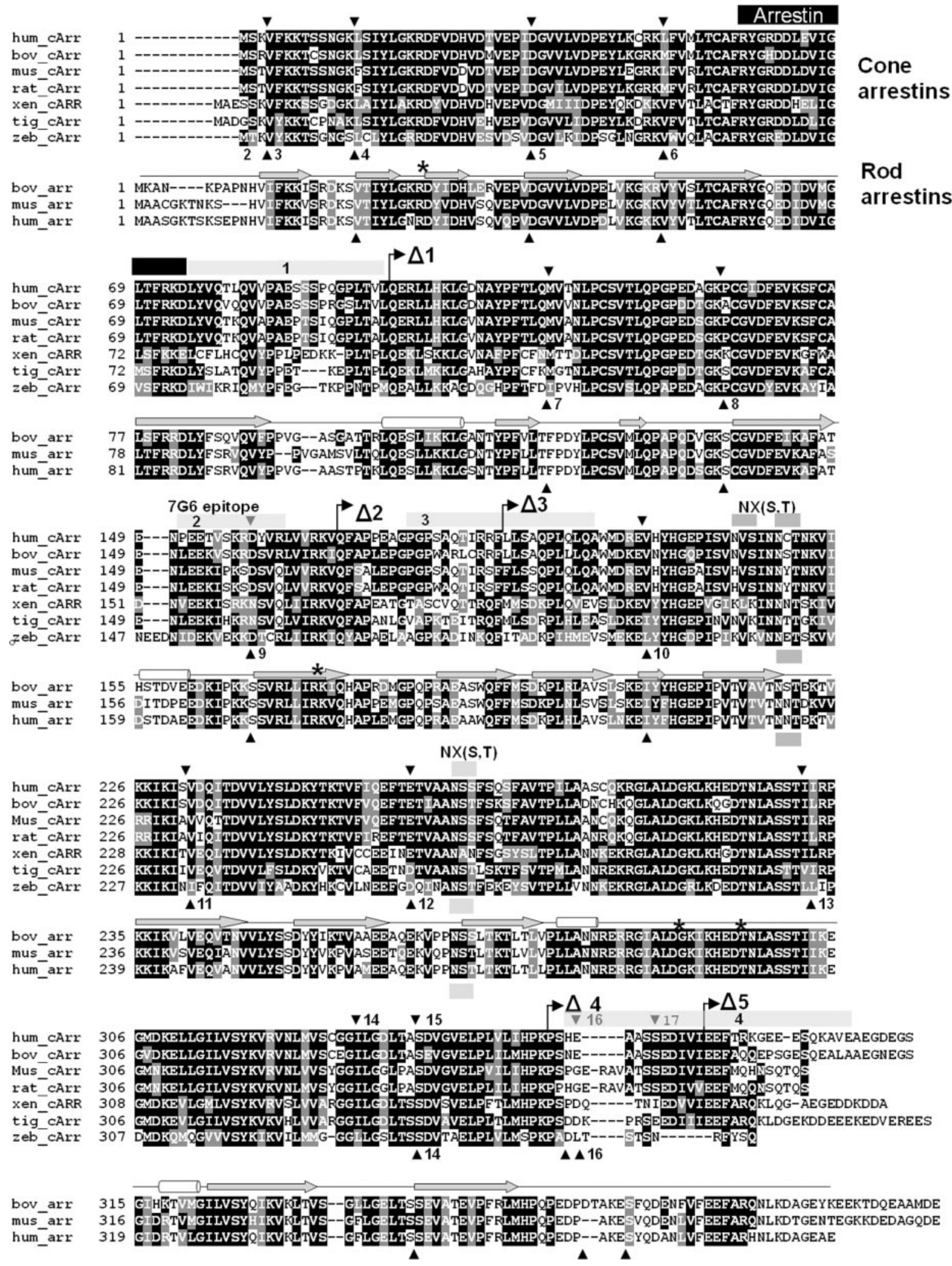

Figure 5. Determination of the 7G6 epitope. Amino acid sequence alignment of rod (Arr) and cone arrestins (cArr). Black triangles, above and below the human and zebrafish sequences, respectively, indicate positions of introns in the gene sequences. Black arrows: truncated areas in mutants of human cone arrestin $(\Delta 1-\Delta 5)$. Shaded bars 1 to 4 : divergent areas. The three-dimensional structure of rod arrestin is indicated above the bovine rod arrestin sequence. Large arrows, $\beta$ sheets; cylinders, $\alpha$-helices.

containing the phosphorylation sites acts as a switch to induce a conformational change in arrestins that enables binding to the receptor. ${ }^{28,37} \mathrm{~A}$ similar model for arrestin/receptor inter- action was proposed in solution on the basis of small angle $x$-ray scattering experiments. ${ }^{38}$ Rod arrestin's binding to the receptor is sensitive to alternative splicing and missense muta- 

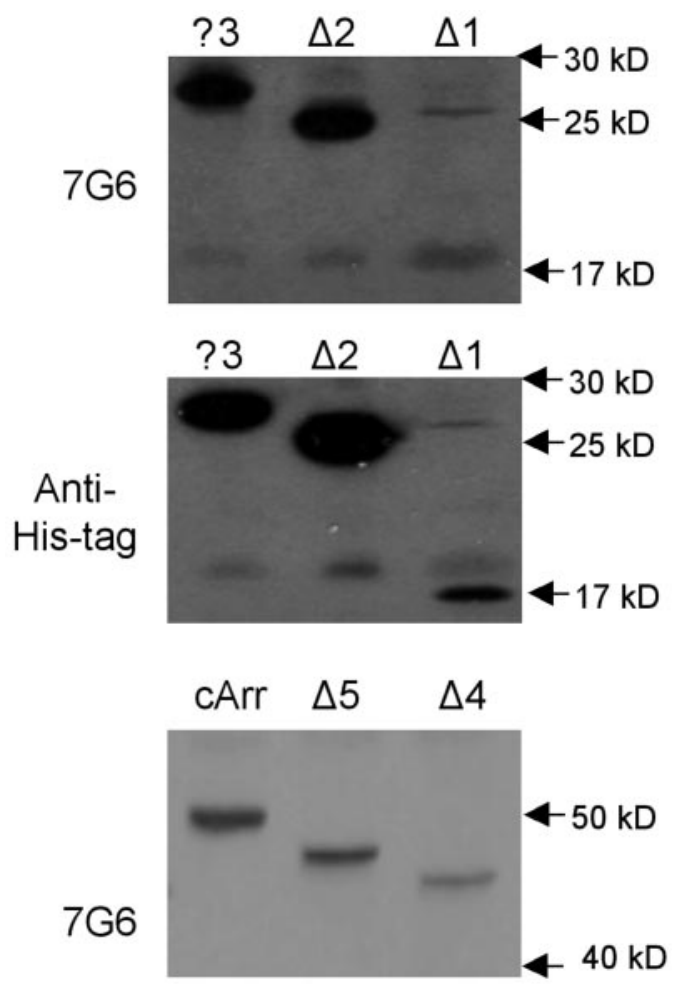

FiguRE 6. Analysis of deletion mutants by Western blot analysis. The human recombinant His-tagged $\mathrm{X}$-arrestin and its deletion mutants $\Delta 1$ to $\Delta 5$ were run on $10 \%$ SDS-PAGE gel and transferred to nitrocellulose membrane. Top: His-tagged mutants $\Delta 1$ to $\Delta 3$ were probed with mAb 7G6 (1:150 dilution). Middle: same mutants probed with anti-His-tag antibody. Note that the $\Delta 1$ mutant was expressed and immunopositive with anti-His-tag antibody, but negative with 7 G6. Bottom: mutant $\Delta 4$ and $\Delta 5$ probed with $\mathrm{mAb} 7 \mathrm{G} 6$.

tions. A bovine arrestin splice variant (p44) without the Cterminal peptide encoded by the last exon can bind to unphosphorylated rhodopsin. ${ }^{39}$ Arrestin mutant R175Q binds to activated phosphorylated and unphosphorylated rhodopsin, and Arr (R175E) binds to bleached rhodopsin from which the C-terminal phosphorylation sites have been removed. ${ }^{40}$ Other residues (D30, R175, D296, and D303 based on the bovine arrestin sequence) have been identified that enable binding to nonphosphorylated rhodopsin. ${ }^{37}$ The mutant arrestins are presumably switched into the active conformation in the absence of rhodopsin phosphorylation. All these residues are well conserved in cone arrestins (Fig. 5, asterisks), suggesting a similar functional role of cone arrestin in quenching the cone cascade. The uniform distribution of cone arrestin throughout the cell indicates that it diffuses freely in the cytoplasm. We cannot exclude that cone arrestin may have additional functions unrelated to quenching the cone cascade.

Malfunctioning in receptor phosphorylation (in which there is no quenching of the cascade) has been linked to cardiac disease in transgenic animal models ${ }^{41}$ and to congenital stationary night blindness in humans. ${ }^{42,43}$ Patients with naturally occurring mutations in rod arrestin have Oguchi disease, a form of inherited stationary night blindness. ${ }^{44}$ In arrestinknockout mice, photoresponses are prolonged, consistent with the absence of quenching. ${ }^{45}$ In addition, it has been demonstrated that excessive light can cause rod cell death in pigmented rod arrestin-knockout mice. ${ }^{46-48}$ In human, the cArr gene is located on the long arm of the $\mathrm{X}$ chromosome near the centromere (Xcen-Xq22). ${ }^{25}$ Because cone arrestin's presumed quenching capacity must be vital for proper cone func- tioning, its gene is considered a candidate gene for cone dystrophies or macular degenerations. No defects in the cone arrestin gene have so far been linked to human retinal dystrophies. In preliminary experiments, we screened three patients with X-linked cone disease for disease-causing mutations, but no mutation was identified (results not shown).

The movement of arrestins and transducin subunits in rods and cones is an interesting phenomenon representing a novel mechanism of light-dark adaptation. In light, arrestins accumulate in the outer segments to quench the phototransduction cascades. Rod transducin subunits, in contrast, move in the opposite direction ${ }^{24,49,50}$ to desensitize the cascade. The traffic from the inner to the outer segments must pass through a narrow interconnecting cilium in both rods and cones. In Kif3A conditional knockout mice, arrestin and rhodopsin transport, but not transducin transport, was shown to be dependent on kinesin II, a ubiquitous molecular motor ${ }^{51}$ present in the cilium. Unresolved questions include the mechanisms that trigger the relocalization of these components and exactly which motors and motor subunits are involved in translocation.

\section{Acknowledgments}

The authors thank Jason Chen and Kim Howes for critical reading of the manuscript.

\section{References}

1. Wikler KC, Rakic P, Bhattacharyya N, MacLeish PR. Early emergence of photoreceptor mosaicism in the primate retina revealed by a novel cone-specific monoclonal antibody. J Comp Neurol. 1997;377:500-508.

2. Chen X, Wikler KC, MacLeish PR. Cone properties of retinal margin cells in the monkey (Macaca mulatta). Invest Ophthalmol Vis Sci. 2000;41:2019-202.

3. John SK, Smith JE, Aguirre GD, Milam AH. Loss of cone molecular markers in rhodopsin-mutant human retinas with retinitis pigmentosa. Mol Vis. 2000;6:204-215.

4. Swain PK, Hicks D, Mears AJ, et al. Multiple phosphorylated isoforms of NRL are expressed in rod photoreceptors. J Biol Chem. 2001;276:36824-36830.

5. Nathans J, Piantanida TP, Eddy RL, Shows TB, Hogness DS. Molecular genetics of inherited variation in human color vision. Science. 1986;232:203-232

6. Lerea CL, Bunt-Milam AH, Hurley JB. Alpha transducin is present in blue-, green-, and red-sensitive cone photoreceptors in the human retina. Neuron. 1989;3:367-376.

7. Li T, Volpp K, Applebury ML. Bovine cone photoreceptor cGMP phosphodiesterase structure deduced from a cDNA clone. Proc Natl Acad Sci USA. 1990;87:293-297.

8. Wissinger B, Muller F, Weyand I, et al. Cloning, chromosomal localization and functional expression of the gene encoding the alpha-subunit of the cGMP-gated channel in human cone photoreceptors. Eur J Neurosci. 1997;9:2512-2521.

9. Gerstner A, Zong X, Hofmann F, Biel M. Molecular cloning and functional characterization of a new modulatory cyclic nucleotidegated channel subunit from mouse retina. J Neurosci. 2000;20: $1324-1332$.

10. Howes K, Bronson JD, Dang YL, et al. Gene array and expression of mouse retina guanylate cyclase activating proteins 1 and 2 . Invest Ophthalmol Vis Sci. 1998;39:867-875.

11. Liu X, Seno K, Nishizawa Y, et al. Ultrastructural localization of retinal guanylate cyclase in human and monkey retinas. Exp. Eye Res. 1994;59:761-768.

12. Zhang $\mathrm{K}$, Howes KA, He W, et al. Structure, alternative splicing, and expression of the human RGS9 gene. Gene. 1999;240:23-34.

13. Zhao X, Huang J, Khani SC, Palczewski K. Molecular forms of human rhodopsin kinase (GRK1). J Biol Chem. 1998;273:51245131.

14. McGinnis JF, Stepanik PL, Baehr W, Subbaraya I, Lerious V. Cloning and sequencing of the $23 \mathrm{kD}$ mouse photoreceptor cell-specific protein. FEBS Lett. 1992;302:172-176. 
15. Milam AH, Dacey DM, Dizhoor AM. Recoverin immunoreactivity in mammalian cone bipolar cells. Vis Neurosci. 1993;10:1-12.

16. Mangini NJ, Pepperberg DR. Immunolocalization of $48 \mathrm{~K}$ in rod photoreceptors: light and ATP increase OS labeling. Invest Ophthalmol Vis Sci. 1988;29:1221-1234.

17. Whelan JP, McGinnis JF. Light-dependent subcellular movement of photoreceptor proteins. J Neurosci Res. 1988;20:263-270.

18. Zhu X, Li A, Brown B, Weiss ER, Osawa S, Craft CM. Mouse cone arrestin expression pattern: light induced translocation in cone photoreceptors. Mol Vis. 2002;8:462-471.

19. Cuenca N, Lopez S, Howes K, Kolb H. The localization of guanylyl cyclase-activating proteins in the mammalian retina. Invest $O p h$ thalmol Vis Sci. 1998;39:1243-1250.

20. Chiquet C, Dkhissi-Benyahya O, Chounlamountri N, Szel A, DeGrip WJ, Cooper HM. Characterization of calbindin-positive cones in primates. Neuroscience. 2002;115:1323-1333.

21. Christakos S, Barletta F, Huening M, Kohut J, Raval-Pandya M. Activation of programmed cell death by calcium: protection against cell death by the calcium binding protein, calbindin-D28K. In: Pochet R, ed. Calcium. The Molecular Basis of Calcium Action in Biology and Medicine. Dordrecht, The Netherlands: Kluwer Academic Publishers; 2000:259-275.

22. Akerstrom B, Brodin T, Reis K, Bjorck L. Protein G: a powerful tool for binding and detection of monoclonal and polyclonal antibodies. J Immunol. 1985;135:2589-2592.

23. McGinnis JF, Matsumoto B, Whelan JP, Cao W. Cytoskeleton participation in subcellular trafficking of signal transduction proteins in rod photoreceptor cells. J Neurosci Res. 2002;67:290-297.

24. Sokolov M, Lyubarsky AL, Strissel KJ, et al. Massive light-driven translocation of transducin between the two major compartments of rod cells: a novel mechanism of light adaptation. Neuron. 2002;34:95-106.

25. Sakuma $\mathrm{H}$, Murakami A, Fujimaki T, Inana G. Isolation and characterization of the human X-arrestin gene. Gene. 1998;224:87-95.

26. Zhu X, Ma B, Babu S, Murage J, Knox BE, Craft CM. Mouse cone arrestin gene characterization: promoter targets expression to cone photoreceptors. FEBS Lett. 2002;524:116-122.

27. Craft CM, Whitmore DH. The arrestin superfamily: cone arrestins are a fourth family. FEBS Lett. 1995;362:247-255.

28. Hirsch JA, Schubert C, Gurevich VV, Sigler PB. The 2.8 A crystal structure of visual arrestin: a model for arrestin's regulation. Cell. 1999;97:257-269.

29. Shichi H, Koch KA. The sugar moiety of bovine S-antigen. Curr Eye Res. 1990;9(suppl):103-109.

30. Palczewski K, Saari JC. Activation and inactivation steps in the visual transduction pathway. Curr Opin Neurobiol. 1997;7:500504.

31. Craft CM, Whitmore DH, Wiechmann AF. Cone arrestin identified by targeting expression of a functional family. J Biol Chem. 1994; 269:4613-4619.

32. Chen CK, Burns ME, Spencer M, et al. Abnormal photoresponses and light-induced apoptosis in rods lacking rhodopsin kinase. Proc Natl Acad Sci USA. 1999;96:3718-3722.

33. Chen CK, Zhang K, Church-Kopish J, et al. Characterization of human GRK7 as a potential cone opsin kinase. Mol Vis. 2001;7: 305-313.
34. Weiss ER, Ducceschi MH, Horner TJ, Li A, Craft CM, Osawa S. Species-specific differences in expression of G-protein-coupled receptor kinase (GRK) 7 and GRK1 in mammalian cone photoreceptor cells: implications for cone cell phototransduction. J Neurosci. 2001;21:9175-9184.

35. Smith WC, Gurevich EV, Dugger DR, et al. Cloning and functional characterization of salamander rod and cone arrestins. Invest $O p h$ thalmol Vis Sci. 2000;41:2445-2455.

36. Maeda T, Ohguro H, Sohma H, et al. Purification and characterization of bovine cone arrestin (cArr). FEBS Lett. 2000;470:336-340.

37. Vishnivetskiy SA, Paz CL, Schubert C, Hirsch JA, Sigler PB, Gurevich VV. How does arrestin respond to the phosphorylated state of rhodopsin? J Biol Chem. 1999;274:11451-11454.

38. Shilton BH, McDowell JH, Smith WC, Hargrave PA. The solution structure and activation of visual arrestin studied by small-angle X-ray scattering. Eur J Biochem. 2002;269:3801-3809.

39. Pulvermüller A, Maretzki D, Rudnicka-Nawrot M, Smith WC, Palczewski K, Hofmann KP. Functional differences in the interaction of arrestin and its splice variant, $\mathrm{p}^{44}$, with rhodopsin. Biochemistry. 1997;36:9253-9260.

40. Gray-Keller MP, Detwiler PB, Benovic JL, Gurevich VV. Arrestin with a single amino acid substitution quenches light-activated rhodopsin in a phosphorylation-independent fashion. Biochemistry. 1997;36:7058-7063.

41. Koch WJ, Rockman HA, Samama P, et al. Cardiac function in mice overexpressing the beta-adrenergic receptor kinase or a beta ARK inhibitor. Science. 1995;268:1350-1353.

42. Cideciyan AV, Zhao XY, Nielsen L, Khani SC, Jacobson SG, Palczewski K. Null mutation in the rhodopsin kinase gene slows recovery kinetics of rod and cone phototransduction in man. Proc Natl Acad Sci USA. 1998;95:328-333.

43. Yamamoto S, Sippel KC, Berson EL, Dryja TP. Defects in the rhodopsin kinase gene in the Oguchi form of stationary night blindness [see comments]. Nat Genet. 1997;15:175-178.

44. Fuchs S, Nakazawa M, Maw M, Tamai M, Oguchi Y, Gal A. A homozygous 1-base pair deletion in the arrestin gene is a frequent cause of Oguchi disease in Japanese. Nat Genet. 1995;10:360 -362.

45. Xu J, Dodd RL, Makino CL, Simon MI, Baylor DA, Chen J. Prolonged photoresponses in transgenic mouse rods lacking arrestin. Nature. 1997;389:505-509.

46. Chen J, Simon MI, Matthes MT, Yasumura D, LaVail MM. Increased susceptibility to light damage in an arrestin knockout mouse model of Oguchi disease (stationary night blindness). Invest Ophthalmol Vis Sci. 1999;40:2978-2982.

47. Hao W, Wenzel A, Obin MS, et al. Evidence for two apoptotic pathways in light-induced retinal degeneration. Nat Genet. 2002; 32:254-260.

48. Jacobson SG, McInnes RR. Blinded by the light. Nat Genet. 2002; 32:215-216.

49. Brann MR, Cohen LV. Diurnal expression of transducin mRNA and translocation of transducin in rods of rat retina. Science. 1987;235: 585-587.

50. Philp NJ, Chang W, Long K. Light-stimulated protein movement in rod photoreceptor cells of the rat retina. FEBS Lett. 1987;225:127132.

51. Marszalek JR, Liu X, Roberts EA, et al. Genetic evidence for selective transport of opsin and arrestin by kinesin-II in mammalian photoreceptors. Cell. 2000;102:175-187. 